\title{
Flexible interwoven termini determine the thermal stability of thermosomes
}

\author{
Kai Zhang ${ }^{1,3}$, Li Wang ${ }^{2}$, Yanxin Liư ${ }^{4}$, Kwok-Yan Chan ${ }^{4}$, Xiaoyun Pang ${ }^{1}$, Klaus Schulten ${ }^{4}$, Zhiyang Dong ${ }^{2}$, \\ Fei Sun ${ }^{1 \mathrm{~d}}$ \\ ${ }^{1}$ National Laboratory of Biomacromolecules, Institute of Biophysics, Chinese Academy of Sciences, Beijing 100101, China \\ ${ }^{2}$ The State Key Laboratory of Microbial Resources, Institute of Microbiology, Chinese Academy of Sciences, Beijing 100101, \\ China \\ ${ }^{3}$ University of Chinese Academy of Sciences, Beijing 100049, China \\ ${ }^{4}$ Beckman Institute and Department of Physics, University of Illinois at Urbana-Champaign, Urbana, IL 61801, USA \\ $\triangle$ Correspondence: feisun@ibp.ac.cn
}

Received April 6, 2013 Accepted April 26, 2013

\begin{abstract}
Group II chaperonins, which assemble as double-ring complexes, assist in the refolding of nascent peptides or denatured proteins in an ATP-dependent manner. The molecular mechanism of group II chaperonin assembly and thermal stability is yet to be elucidated. Here, we selected the group II chaperonins (cpn- $\alpha$ and cpn- $\beta$ ), also called thermosomes, from Acidianus tengchongensis and investigated their assembly and thermal stability. We found that the binding of ATP or its analogs contributed to the successful assembly of thermosomes and enhanced their thermal stabilities. Cpn- $\beta$ is more thermally stable than cpn- $\alpha$, while the thermal stability of the hetero thermosome cpn- $\alpha \beta$ is intermediate. Cryo-electron microscopy reconstructions of cpn- $\alpha$ and cpn- $\beta$ revealed the interwoven densities of their non-conserved flexible N/C-termini around the equatorial planes. The deletion or swapping of their termini and $\mathrm{pH}$-dependent thermal stability assays revealed the key role of the termini electrostatic interactions in the assembly and thermal stability of the thermosomes.
\end{abstract}

KEYWORDS group II chaperonin, thermosome, thermal stability, self-assembly, flexible terminus

\section{INTRODUCTION}

Chaperonins are homo- or hetero-oligomeric double-ring complexes that use an ATP-dependent mechanism to sequester non-native proteins in their central cavity and mediate folding of the substrate protein into its biologically active state (Ellis, 2006). Each subunit of chaperonin contains a globular apical domain to which unfolded substrate protein binds, an intermediate domain, and an equatorial domain that binds nucleotide and forms the interface between the two rings (Ditzel et al., 1998; Xu et al., 1997). Based on the structures and sequences, chaperonins are conventionally divided into two subfamilies (Kim et al., 1994). Group I chaperonins exist in eubacteria and endosymbiotic organelles (mitochondria and chloroplasts), as typified by the well-studied E. coli chaperonin GroEL (Bukau and Horwich, 1998; Ranson et al., 2001; Ranson et al., 2006; Clare et al., 2009; Clare et al., 2012;), and generally consist of double homo-heptameric ring arrangements. Group II chaperonins are found in archaea (Phipps et al., 1991) and eukaryotic cytosol (Frydman et al., 1992; Gao et al., 1992) and consist of a double hetero (or homo)-octameric or nonameric ring. Unlike group I chaperonins, which require the cofactor GroES (Tilly et al., 1981) to enclose the cage, a subunit of group II chaperonins contains an additional helical protrusion domain that acts as a built-in lid to seal off the central cavity, in which the folding of the substrate takes place (Ditzel et al., 1998; Klumpp et al., 1997).

Archaeal group II chaperonins are also referred as thermosomes due to their extreme thermal stability (Bigotti and Clarke, 2008). The subunit composition of thermosomes varies at different growth temperatures (Izumi et al., 2001; Yoshida et al., 2001; Kagawa et al., 2003), a result that has been suggested to be correlated with the various thermal stabilities of the subunits (Yoshida et al., 2002; Sahlan et al., 2009). The thermal stability of the thermosome cpn- $\beta$ in Thermococcus sp. strain KS-1 (T. KS-1) was found to be higher than that of cpn- $\alpha$ 

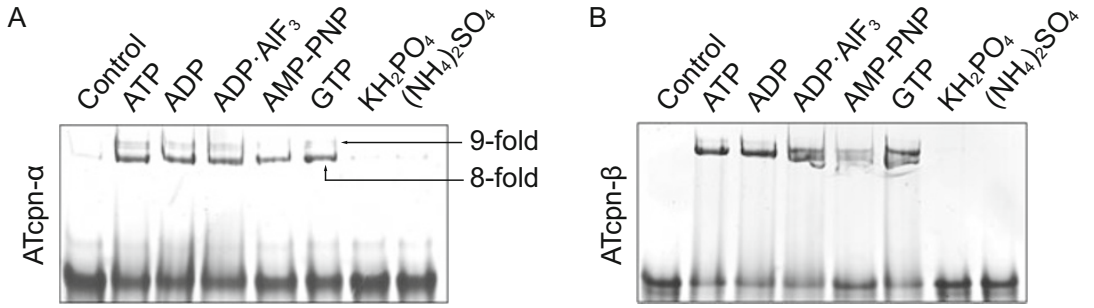

Figure 1. AT thermosomes assembling under different experimental conditions. (A) AT cpn- $\alpha$. (B) AT cpn- $\beta$. The lower bands represent monomers, and the upper bands represent assemblies.
(Yoshida et al., 2002). It has been suggested that the C-terminal residues play an important role in maintaining the thermal stability of thermosomes (Yoshida et al., 2006). A recent study of the thermosome from Pyrococcus furiosus (Pf) revealed that its C-terminal EK-rich motif may be responsible for its extreme thermal stability at $\sim 100^{\circ} \mathrm{C}$ (Luo and Robb, 2011). Despite the above biochemical observations, it is not clear how the Cterminal charged residues dominantly determine the thermal stability of thermosomes.

Here, we used the thermosomes from Acidianus tengchongensis (AT) strain S5 as a model system to investigate the origin of its thermal stability using biochemical and structural assays. The archaea AT grows in the Tengchong acidothermal springs in Southwestern China at an optimal growth temperature of $70^{\circ} \mathrm{C}$ and a $\mathrm{pH}$ of 2.5 (He et al., 2004). Previously, we discovered two types of thermosomes (cpn- $\alpha$ and cpn- $\beta$ ) from this archaea that share $51 \%$ sequence identity. We also showed that recombinant cpn- $\alpha$ predominately assembles into an eight-fold double-ring structure and that recombinant cpn- $\beta$ assembles into a nine-fold structure (Wang et al., 2010). In the current study, we further investigated the assembly and thermal stability of these two thermosomes. We found that the structurally flexible $\mathrm{N}$ - and $\mathrm{C}$-termini interweave together through electrostatic interactions in the center of the chamber and that such interwoven termini are required for the successful assembly of thermosomes and predominately determine their thermal stability. Furthermore, we also addressed the important role of nucleotide binding in the assembly and thermal stability of the thermosomes. Our study provides essential examples for the importance of termini residues for protein assembly and thermal stability.

\section{RESULTS}

Nucleotide binding is necessary and sufficient to induce thermosome assembly

We incubated purified recombinant AT cpn- $\alpha$ and $c p n-\beta$ monomers with different nucleotides (ATP, ADP, ADP•AIF 3 , AMPPNP and GTP), sulfate salt or phosphate salt at $37^{\circ} \mathrm{C}$ for $8 \mathrm{~h}$. Then, we utilized native polyacrylamide gel electrophoresis (PAGE) to determine whether the monomers assemble into thermosomes. We found that all nucleotides could efficiently induce the assembly of thermosomes for both cpn- $\alpha$ (Fig. 1A) and cpn- $\beta$ (Fig. 1B). However, without added nucleotides, both cpn- $\alpha$ and cpn- $\beta$ failed to assemble into thermosomes
(Fig. 1). These results indicate that nucleotide binding is necessary for the thermosome monomers to adopt the appropriate conformation and oligomerize. Previous work has shown that free sulfate acid or phosphate acid could bind to the P-loop of the thermosome subunit (Shomura et al., 2004). However, such binding may not induce sufficient conformational changes for thermosome assembly. Our results showed that AT cpn- $\alpha$ and cpn- $\beta$ still remain as monomers after incubation with ammonium sulfate or sodium dihydrogen phosphate (Fig. 1). Furthermore, not only ATP but also non-hydrolyzed ATP analogs (ADP, ADP•AlF 3 and AMP-PNP) and even GTP could induce the assembly of thermosomes, indicating that ATP hydrolysis is not required for thermosome assembly. Based on our results, we postulate that it is not the binding of ATP's phosphate head but the binding of ATP's adenosine tail that induces the successful assembly of thermosomes.

\section{Distinct thermal stability of the thermosomes cpn- $\alpha$ and cpn- $\beta$}

Thermo-shift assays showed that cpn- $\alpha$ and cpn- $\beta$ monomers are thermally stable at high temperatures, with similar denaturing temperatures of $88.7^{\circ} \mathrm{C}$ and $87.4^{\circ} \mathrm{C}$, respectively (Fig. 2A), which are higher than the growth temperature $\left(55^{\circ} \mathrm{C}-80^{\circ} \mathrm{C}\right)$ of their natural host, AT (He et al., 2004). However, the assembled thermosomes also need sufficient thermal stability to survive in the natural environment. We utilized a native PAGE approach to study the dissociation of the thermosomes cpn- $\alpha$ and cpn- $\beta$ at different temperatures.

We heated cpn- $\alpha$ for $10-90$ min at $45^{\circ} \mathrm{C}, 50^{\circ} \mathrm{C}, 55^{\circ} \mathrm{C}$, and $60^{\circ} \mathrm{C}$ before subjecting the protein to native PAGE. We found that cpn- $\alpha$ is relatively stable at $45^{\circ} \mathrm{C}$ and begins to dissociate at higher temperatures (Fig. S1). Thirty minutes of heating was sufficient to reach an equilibrium between the oligomers and monomers at different temperatures (Fig. 2B). Therefore, we heated all the thermosomes for 30 min before investigating their dissociations at different temperatures (Fig. 2C-E). The cpn- $\alpha$ protein exhibits two major bands representing its 8-fold and 9-fold assemblies, which have been previously reported (Huo et al., 2010). Without ATP, the 8-fold cpn- $\alpha$ thermosome dissociated completely when heated to $65.8^{\circ} \mathrm{C}$ (the critical dissociation temperature $T_{d}$ ), and the 9-fold assembly is a bit more stable than the 8-fold assembly, with a $T_{d}$ of $68.8^{\circ} \mathrm{C}$ (Fig. $2 \mathrm{C}$ ). The cpn- $\beta$ is more thermally stable than cpn- $\alpha$, with $a T_{d}$ of up to $85.1^{\circ} \mathrm{C}$ (Fig. 2D). The hetero thermosome cpn- $\alpha \beta$, which is assembled from both cpn- $\alpha$ and cpn- $\beta$ (Wang et al., 


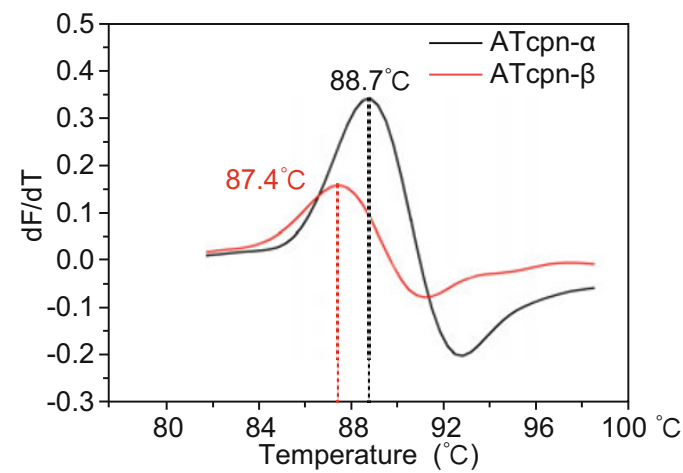

C

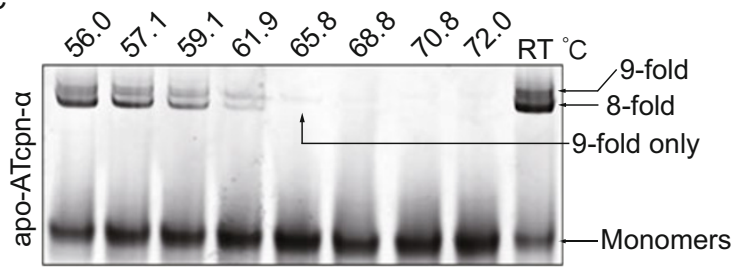

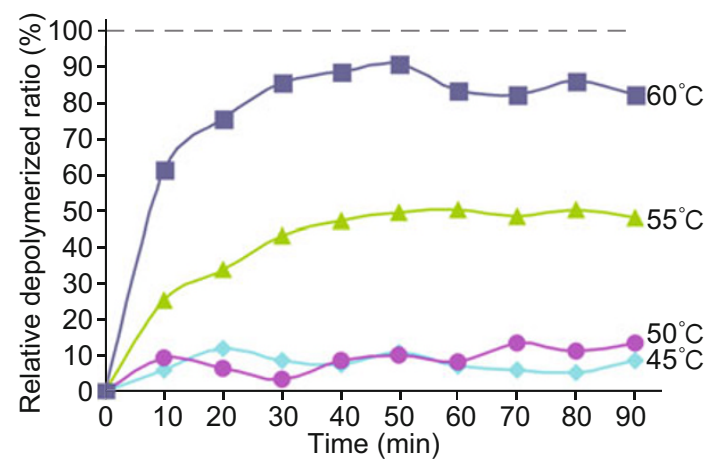

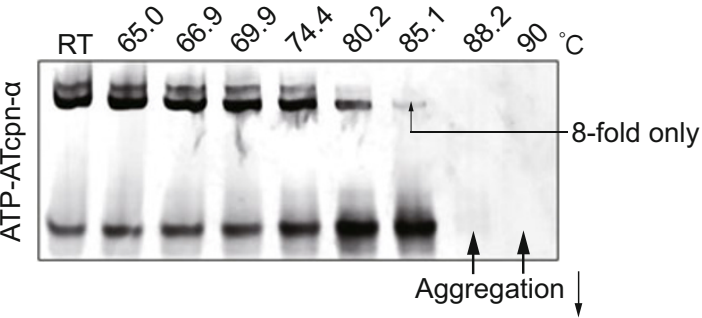

$E$
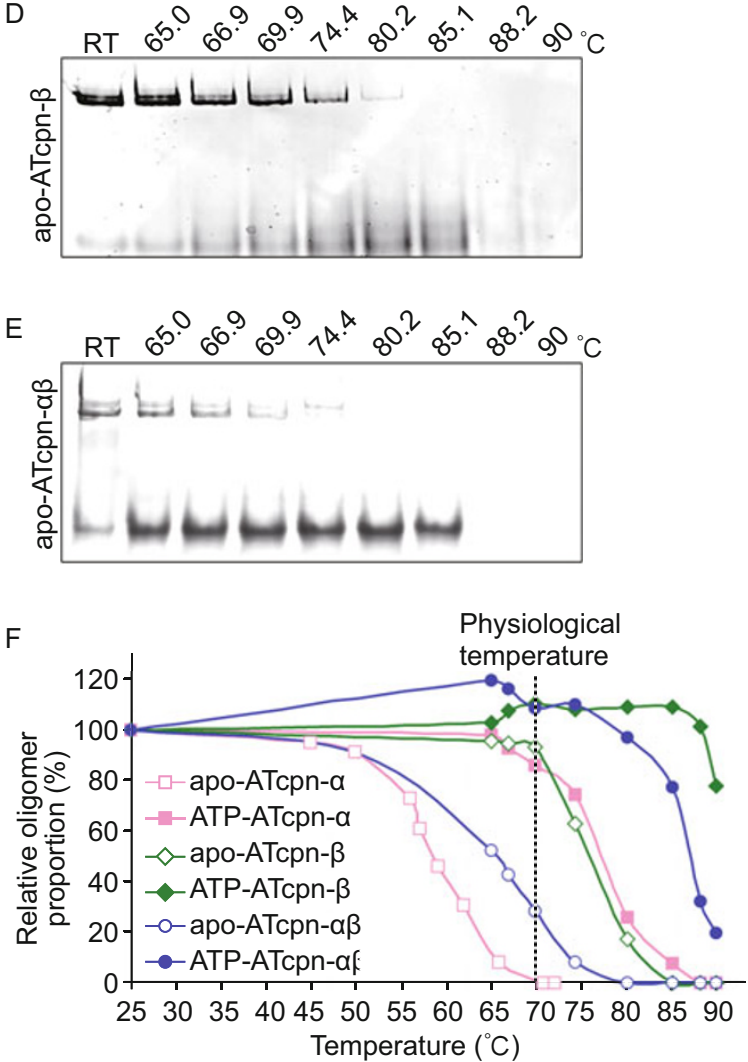

D

$\mathrm{F}$

2010), has a $T_{d}$ of $74.4^{\circ} \mathrm{C}$, which is between that of cpn- $\alpha$ and cpn- $\beta$ (Fig. 2E).

Furthermore, we found that the thermo-stabilities of thermosomes are greatly enhanced by adding ATP. In the presence of ATP, the $T_{d}$ of 8-fold and 9-fold cpn- $\alpha$ increased to $88.2^{\circ} \mathrm{C}$ and $85.1^{\circ} \mathrm{C}$, respectively (Fig. $2 \mathrm{C}$ ), and interestingly, the 8-fold assembly became more stable than the 9-fold one. The $T_{d}$ of both cpn- $\beta$ and cpn- $\alpha \beta$ also increased to over $90^{\circ} \mathrm{C}$ in the ATP binding state (Fig. 2D and 2E). It should be noted that both the cpn- $\alpha$ and cpn- $\beta$ monomers became unfolded and aggregated at $88.2^{\circ} \mathrm{C}$ (the monomer bands disappeared above this temperature), which is in accordance with the above 
A

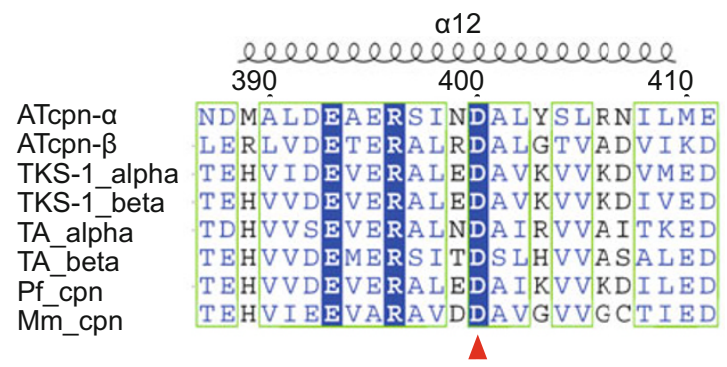

C

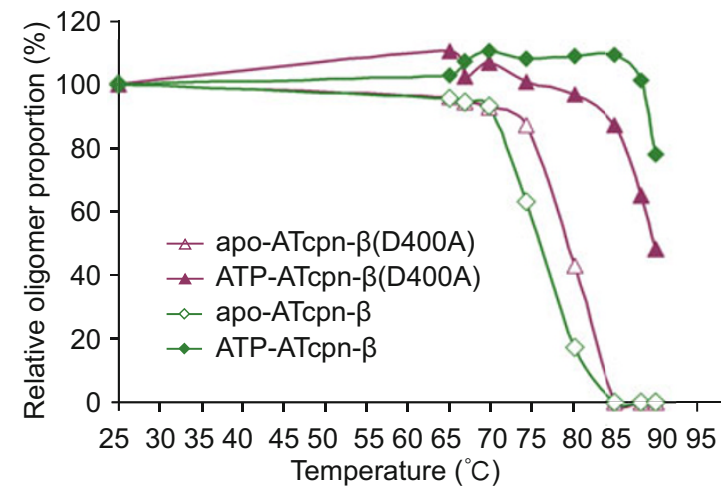

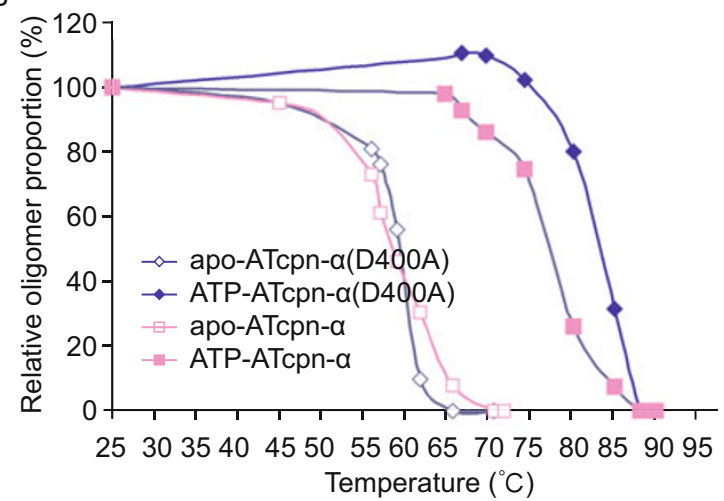

$\mathrm{D}$

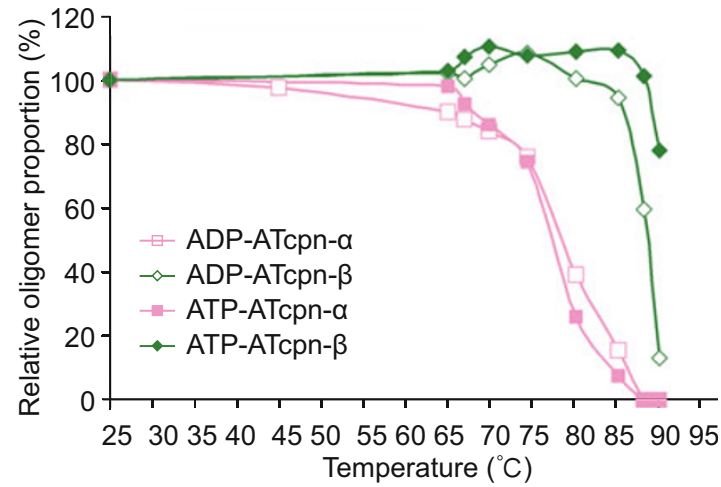

Figure 3. Quantified comparisons of thermal stabilities for wild-type and D400A mutant thermosomes and for the ATP-binding and the ADP-binding states. (A) Sequence alignment of thermosomes around the residue D400. AT, Acidianus tengchongensis; TKS-1, Thermococcus sp. strain KS-1; TA, Thermoplasma acidophilum; Pf, Pyrococcus furiosus; Mm, Methanococcus maripaludis. (B) Thermal stability comparison between the wild-type AT cpn- $\alpha$ and its D400A mutant in both the apo and ATP-binding state. (C) Thermal stability comparison between the wild-type AT cpn- $\beta$ and its D400A mutant in both the apo and ATP-binding state. (D) Thermal stability comparison between the ATP- and ADP-binding states of both AT cpn- $\alpha$ and cpn- $\beta$. All the data for the wild type AT cpn- $\alpha$ and cpn - $\beta$ in apo and ATP-binding states are the same as Fig. 2F. See also Fig. S2.

thermo-shift experiments (Fig. 2A). The results indicate that in an ATP binding state, the interactions between subunits not only significantly stabilize the thermosome assembly but also stabilize the ternary structure within each subunit.

From the native PAGE results, we calculated the percentage of remaining oligomers at different temperatures for each thermosome in their apo and ATP binding states (Fig. 2F). It is obvious that the thermosome cpn- $\beta$ is thermally more stable than cpn- $\alpha$ in both the apo and the ATP binding state.

\section{ATP hydrolysis is not required for the stability of thermosomes}

The thermo-stabilities of all thermosomes, including cpn- $\alpha$, cpn- $\beta$, and cpn- $\alpha \beta$, were significantly enhanced by the presence of ATP. It is possible that ATP hydrolysis but not ATP binding stabilizes the thermosomes. To rule out this possibility, we mutated residue Asp400 to Ala in both cpn- $\alpha$ and cpn- $\beta$. This residue is conserved in all group II chaperonins (Fig. $3 A$ ) and has been reported to play a key role in deprotonation during
ATP hydrolysis (Ditzel et al., 1998). The mutant D400A inhibits ATP hydrolysis. In the presence or absence of ATP, the D400A mutants of both cpn- $\alpha$ and cpn- $\beta$ exhibited the same thermostabilities as their wild-type counterparts (Fig. $3 B$ and $3 C$, Fig. S2A and S2B). Furthermore, ADP binding also increased the thermal stability of $c p n-\alpha$ and $c p n-\beta$ to the same extent as ATP binding (Figs. 3D and S2C). We conclude that the binding of ATP or its analogs is sufficient to stabilize the thermosome, while ATP hydrolysis is not required.

\section{Cryo-electron microscopy of the cpn- $\alpha$ and cpn- $\beta$ thermosomes}

To understand the mechanism of the thermo-stabilities of cpn- $\alpha$ and cpn- $\beta$, we determined their $3 \mathrm{D}$ structures by cryo-electron microscopy (cryoEM) (Fig. 4A-D and Table S1). According to the Fourier shell correlation (FSC) threshold at cutoffs of $0.5 / 0.143$, the reconstruction resolutions of 8 -fold and 9-fold cpn- $\alpha$ are 4.9/4.1 $\AA$ and 9.1/7.5 $\AA$, respectively. The highresolution reconstruction of 8 -fold $\mathrm{cpn}-\alpha$ enabled us to build a 


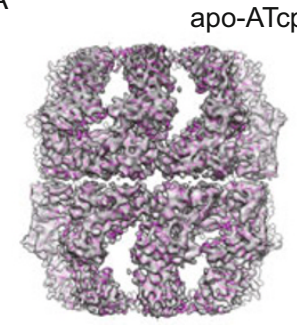

Side view

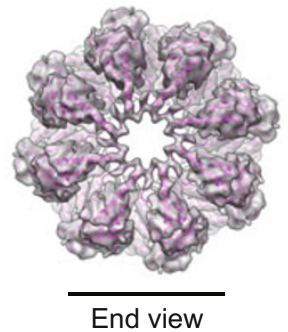

End view

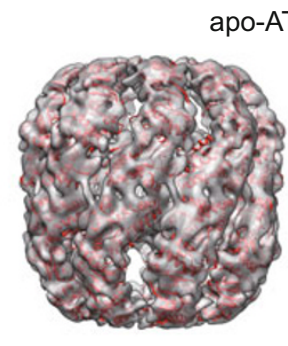

Side view
apo-ATcpn- $\alpha$ (9-fold)

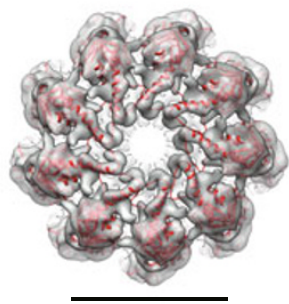

End view

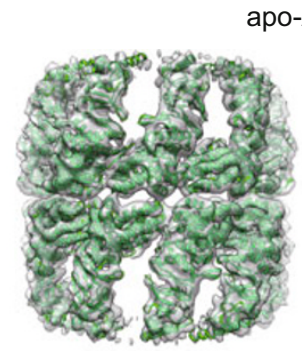

Side view

po-ATcpn- $\beta$

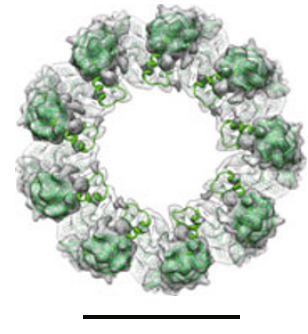

End view

D

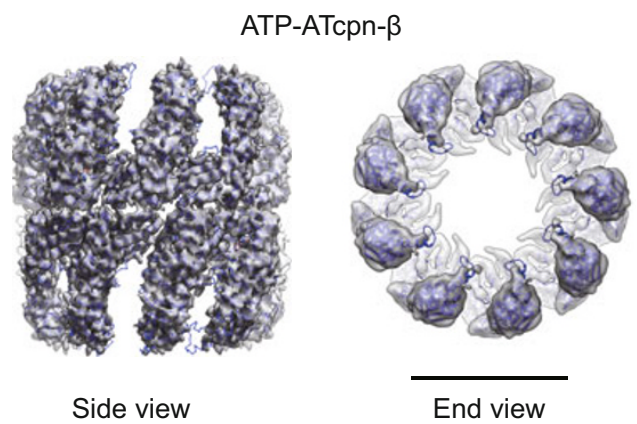

E

apo-ATcpn- $\alpha$ (8-fold)

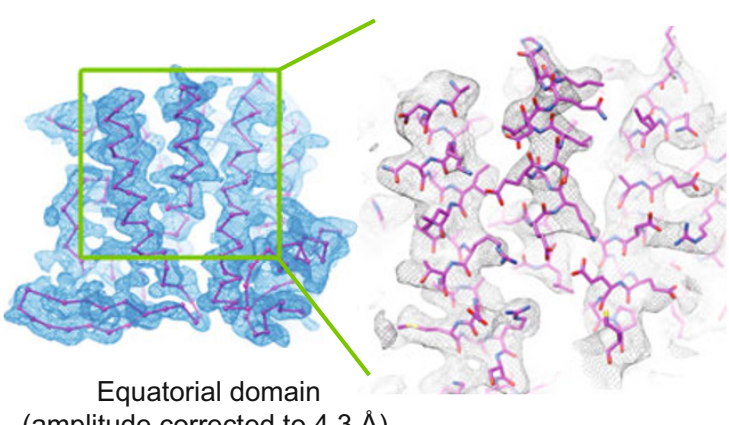

Open Mm-cpn End views
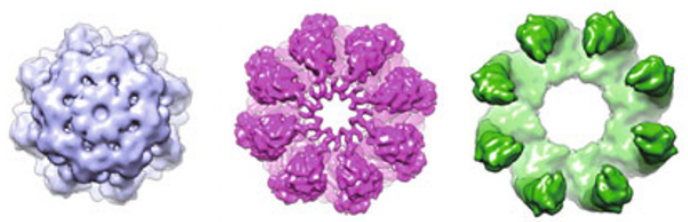

Side views

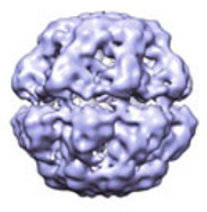

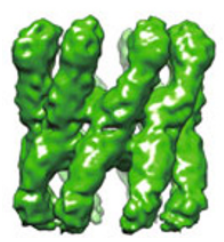

Figure 4. CryoEM reconstructions of AT thermosomes. (A and B) apo AT cpn- $\alpha$ in 8-fold and 9-fold symmetries. (C and D) AT cpn- $\beta$ in apo and ATP-bound states. The end-views of 8 -fold apo cpn- $\alpha$ and ATP-bound cpn- $\beta$ show the maps that are low-pass filtered to a resolution of 8.0 $\AA$. The atomic models are shown in cartoons and fitted into the cryoEM maps with the apo 8-fold cpn- $\alpha$ in magenta, the apo 9-fold cpn- $\alpha$ in red, the apo cpn- $\beta$ in green, and the ATP-bound cpn- $\beta$ in blue. The central densities of these thermosomes are not displayed here due to the small depth of the view. Scale bars for $(A-D)$ are $10 \mathrm{~nm}$. (E) High resolution cryoEM map of apo cpn- $\alpha$. The equatorial cryoEM map is fitted with the backbone of apo cpn- $\alpha$ (left), and the enlarged view from the green rectangle shows that side chains could be discriminated in the density (right). An amplitude correction was applied to $4.3 \AA$ on the final cryoEM map (right). (F) Comparison of the compact structure of apo AT cpn- $\alpha$ (middle, low passed to $8 \AA$ ) with structures of Mm-cpn in the fully closed (left, EMDB code: EMD-1398) and open (right, EMDB code: EMD-1396) states. See also Figs. S3 and S4.

reliable atomic model of cpn- $\alpha$ using the symmetry-restrained molecular dynamics flexible fitting (MDFF) approach (Trabuco et al., 2008; Chan et al., 2011) (Fig. 4E and Fig. S3A-C). Moreover, we obtained the cryoEM reconstructions of $c p n-\beta$ in the apo or ATP-binding state at a resolution of 8.3/6.7 $\AA$ and
6.2/5.0 $\AA$ at FSC $=0.5 / 0.143$, respectively (Fig. 4C and 4D), which was a higher resolution than was used in our previous study (Huo et al., 2010). Atomic structural models of $c p n-\beta$ in both the apo and ATP-binding state were also obtained using symmetry-restrained MDFF (Trabuco et al., 2008; Chan et al., 2011). 
Similar to our previous study (Huo et al., 2010), both apo and ATP-bound cpn- $\beta$ present an open conformation in a cylindrical shape (Fig. 4C and 4D). Only the equatorial domains are involved in the inter-ring and intra-ring contacts, while the intermediate domains and lid-apical domains are disconnected between subunits. In contrast to cpn- $\beta$, the overall structure of cpn- $\alpha$ exhibits a relatively compact conformation that is different from all reported structures of group II chaperonins (Fig. 4A and 4B). Both the equatorial and lid domains of cpn- $\alpha$ contribute to the intra-ring interactions, and the overall shape is intermediate between the fully closed and open structures. The height and width of the 8-fold/9-fold cpn- $\alpha$ are 172/171 $\AA$ and $159 / 172 \AA$, respectively, with the entrance diameter of the chamber only $\sim 38 / 45 \AA$, which is much smaller than any reported open structures. Compared to the structures of 8-fold $\mathrm{Mm}-\mathrm{cpn}$ in the open and fully closed states (Clare et al., 2008; Zhang et al., 2010; Douglas et al., 2011), AT cpn-a has a similar equatorial domain stacking to the open $\mathrm{Mm}-\mathrm{cpn}$, but its intermediate and apical domains have obviously different conformations than both closed and open Mm-cpn (Fig. 4F). The same intra-ring interactions among lids and among equatorial domains are observed in both the 8-fold and 9-fold structures of cpn- $\alpha$ (Fig. 4A and 4B). The structural differences between the subunits in 8-fold and 9-fold cpn- $\alpha$ are minor (Fig. S3D). The only difference between the 8-fold and 9-fold structures of cpn- $\alpha$ is a 5 degree rotation of the adjacent subunit in the 9-fold cpn- $\alpha$ with respect to that in the 8-fold one (Fig. S3D). Therefore, the relatively compact structure of the 8-fold cpn- $\alpha$ is an intrinsic characteristic and is not due to its unique symmetry.

\section{The minor role of the lid domain in the thermal stability of} thermosomes

The structures of cpn- $\alpha$ show that in addition to the equatorial domains, the lids are also involved in intra-ring contacts, which might contribute to the stability of the thermosome. We therefore investigated the role of the lid domain in the thermal stability of cpn- $\alpha$. We constructed a lidless cpn- $\alpha$ mutant by truncating the lid (residues 253-282) and found that in the absence of ATP, this deletion mutant forms fewer oligomers (Fig. S4A). The $T_{d}$ of apo lidless cpn- $\alpha$ slightly decreased to $61.9^{\circ} \mathrm{C}$, while the $T_{d}$ of the ATP-binding lidless cpn- $\alpha$ stayed unchanged. The denaturing temperature of the lidless monomer was slightly decreased, from $88^{\circ} \mathrm{C}$ to $85^{\circ} \mathrm{C}$. These results show that the lid domain can affect the assembly and stability of cpn- $\alpha$. In contrast, the deletion of the lid domain from cpn- $\beta$ did not change its thermal stability (Fig. S4B), although the denaturing temperature of the monomer was also slightly decreased from $88^{\circ} \mathrm{C}$ to $85^{\circ} \mathrm{C}$. Therefore, we can conclude that it is not the lid domain but other structural components that account for the high thermal stability of cpn- $\beta$.

The non-conserved flexible N/C-termini are interwoven in the center of the thermosome chamber

With $51 \%$ sequence identity and more than $70 \%$ sequence ho- mology, the most distinct sequences between cpn- $\alpha$ and cpn- $\beta$ are their non-conserved $\mathrm{N}$ - and C-termini (Fig. 5A). Recent studies have suggested that the thermo-stabilities of group II chaperonins are closely related to their non-conserved Cterminal charged residues (Luo and Robb, 2011). Comparing the C-terminal residues between cpn- $\alpha$ and cpn- $\beta$, there are more charged residues in cpn- $\beta$ ( 8 charged residues between residues 534 and 553 , net charge 0 ) than in cpn- $\alpha$ ( 5 charged residues from 534 to 563 , net charge -1 ), which might explain cpn- $\beta$ 's higher thermal stability. By mapping these terminal residues onto the cryoEM maps of cpn- $\alpha$ and $c p n-\beta$, it is possible to understand how the non-conserved terminal residues affect the thermal stabilities of the thermosomes.

It is well known that both the $\mathrm{N}$ - and C-termini of group II chaperonins contribute to intra-ring interactions by forming anti-parallel stands with the equatorial domain of the adjacent subunit (Ditzel et al., 1998; Shomura et al., 2004). The Nterminus contributes to the first strand, and the C-terminus contributes to the last strand. However, the non-conserved $\mathrm{N}$ - and C-terminal residues before the first strand and after the last strand cannot be traced due to lack of clear densities (Ditzel et al., 1998; Shomura et al., 2004). In a high-resolution cryoEM map of cpn- $\alpha$, we found that the $\mathrm{N}$-terminal residues 14-17 of AT cpn- $\alpha$ form the first stand (Fig. 5B) before the first helix $\alpha 1$, the $\mathrm{C}$-terminal residues 528-532 form the last strand, and there are no traceable densities for the $\mathrm{N}$-terminal first 13 residues and the $\mathrm{C}$-terminal last 31 residues. However, for AT cpn- $\beta$, the residues $23-26$ did not form a strand before the first helix (Fig. 5B), and its N-terminal did not show clear densities; its $\mathrm{C}$-terminal residues did form the last strand from 528 to 532 , and the densities of the last 21 residues were untraceable. Thus, both the $\mathrm{N}$ - and $\mathrm{C}$-termini of thermosomes have flexible residues that do not form stable conformations and cannot be resolved either by our cryoEM study or by previous crystallographic studies (Ditzel et al., 1998; Shomura et al., 2004).

However, when we filtered the cryoEM map of cpn- $\alpha$ to a resolution of $8 \AA$ and computed the difference map by subtracting the fitted MDFF model from the cryoEM map, we observed two pieces of plug-like densities in the middle of the chamber (Fig. $5 \mathrm{C}$ ). For cpn- $\beta$, the difference map revealed that there is one piece of ring-like density in the central position of the thermosome (Fig. 5D). The following analyses revealed that these difference maps account exactly for the un-traced terminal residues of all subunits in both cpn- $\alpha$ or $c p n-\beta$.

First, the total number of untraced residues of the N/C-termini in the entire thermosome is 704 residues for 8 -fold cpn- $\alpha$, 792 residues for 9 -fold $c p n-\alpha$ and 864 residues for $c p n-\beta$. The molecular weight of the total untraced residues is over $100 \mathrm{kDa}$ and is much larger than one entire thermosome subunit ( 60 $\mathrm{kDa}$ ). Such a large mass should contribute to the contrast of each particle projection and cannot be ignored in the final 3D reconstruction. Therefore, the averaging of a large number of particles during reconstruction will yield a mean summation of all possible termini densities that should be positive rather than zero. 
A

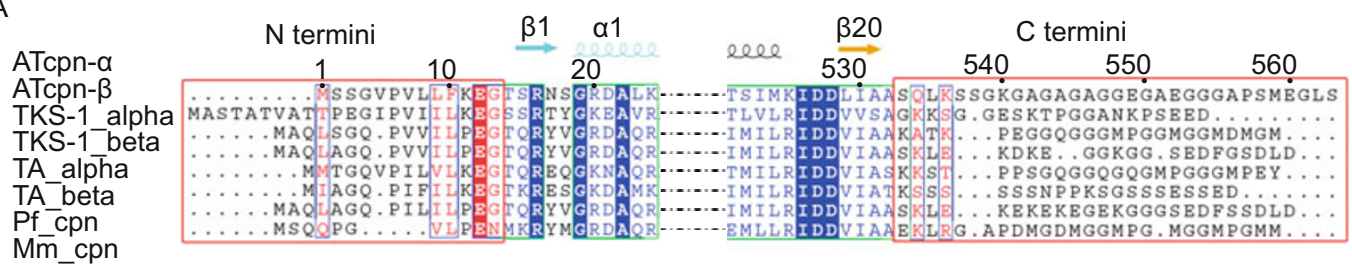

B
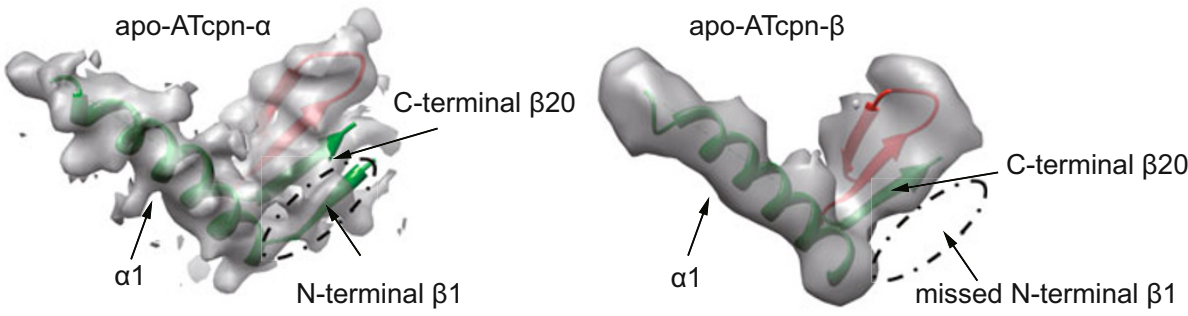

C
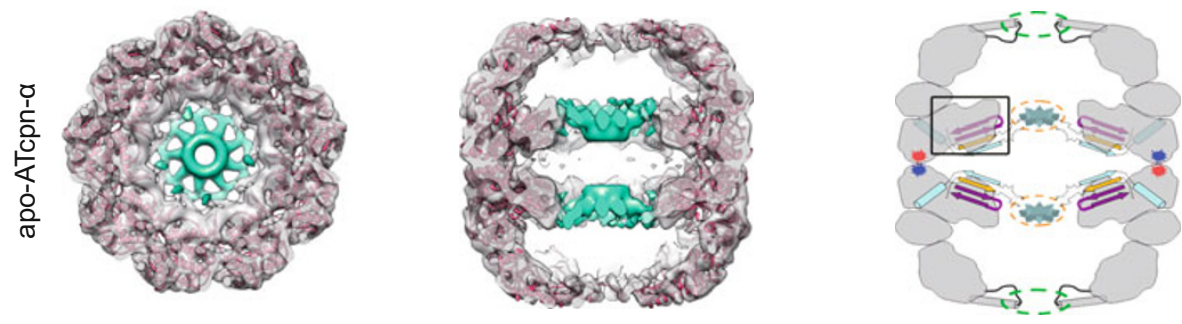

D
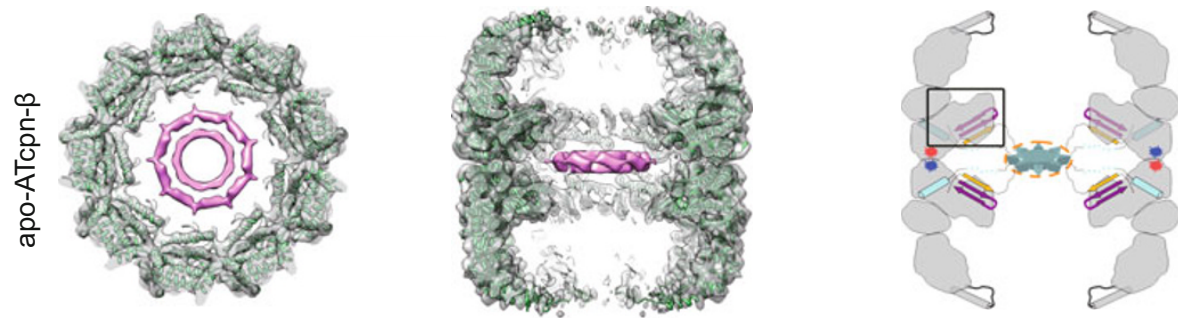

- Inter-ring equatoria domain interaction

Lid domain interaction

Interweaved termini

N-terminal beta-strand

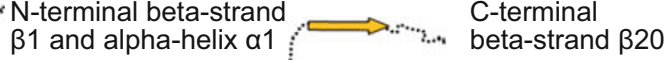

Beta-strands of adjacent subunit

Figure 5. Termini of AT thermosomes. (A) Sequence alignment of different group II chaperonins around the N/C-terminal regions. Nonconserved N/C-termini are highlighted by red boxes. AT, Acidianus tengchongensis; TKS-1, Thermococcus sp. strain KS-1; TA, Thermoplasma acidophilum; Pf, Pyrococcus furiosus; Mm, Methanococcus maripaludis. (B) The enlarged views of the cryoEM maps of the apo AT cpn- $\alpha$ and cpn- $\beta$ around the equatorial regions. The built models are fitted into the maps. The first strand (14-18) in cpn- $\alpha$ is indicated within a black dashed ellipse, and its equivalent position in cpn- $\beta$, where the first strand is missing, is also indicated. (C and D) CryoEM densities of the N/C-termini in apo cpn- $\alpha$ and cpn- $\beta$ at $1.0 \sigma$ counter level. All the structural elements are depicted. See also Fig. S5 and Movies S1 and S2.

Second, one may argue that such extra densities would just be reconstruction noises. However, when we visualize the cryoEM reconstruction of $c p n-\beta$ in a volume rendering manner (Fig. S5A and Movie S1), we can observe all of the density at the same time, including the noise. It is obvious that the central density of cpn- $\beta$ is much stronger than the noise. Furthermore, by masking out the central density, the final refined model of cpn- $\beta$ was filtered to a $25 \AA$ map and then was used to perform the asymmetric reconstruction against the same particle set. As a result, the reconstructed asymmetric map shows clear high density in the central region that cannot be accounted for by noise (Fig. S5B and Movie S2). Further cryoEM analysis of 
the N/C-termini truncated form of cpn- $\beta$ in ATP binding state, showing no extra densities in the central chamber (Figure $\mathrm{S} 5 \mathrm{C}$ ), also ruled out the possibility of the reconstruction noise.

Third, as described previously (Nitsch et al., 1997; Dekker et al., 2011; Munoz et al., 2011), one might consider that the extra densities existing in the center of the chamber could represent a substrate trapped by the thermosome during purification. However, unlike previous studies in which group II chaperonins were purified directly from intact cells, we purified the monomers of AT cpn- $\alpha$ and cpn- $\beta$ and then assembled them into thermosomes. By adopting such a protocol, we avoided any chance of trapping substrate inside the chaperonins.

Finally, the difference maps are located in the center of the thermosome, between the two rings (not in either ring) and in proximity to the subunit terminus. Therefore, we concluded that the extra density in the center of the thermosome is a real signal rather than noise and accounts for the $\mathrm{N}$ - and $\mathrm{C}$-termini of cpn- $\alpha$ and cpn- $\beta$.

Clearly, the termini of the cpn- $\alpha$ upper ring and lower ring interweave separately and form two separated densities. Each plug-like density sits just on the bottom of each folding cage. We refer to this type of interacting termini as A-style termini. However, all the un-traced terminal residues in cpn- $\beta$ gather together and interact with each other to form a ring-like structure between two folding cages. We refer to this type of interacting termini as B-style termini. Whether the termini are of the A-style or the B-style type, they are all interwoven together and form a ring-like structure in the center of the thermosome chamber; thus, we suggested that these interwoven termini are responsible for the assembly and thermal stability of the thermosomes.

The interwoven N/C-termini predominately determine the assembly and thermal stability of the thermosomes

Our cryoEM studies suggested that not only the C-terminal residues but also the $\mathrm{N}$-terminal residues form an interwoven structure in the center and are involved in the determinants of the thermal stability of thermosomes. To confirm this hypothesis, we performed mutagenesis studies by deleting the flexible $\mathrm{N} / \mathrm{C}$-termini or exchanging them between $\mathrm{cpn}-\alpha$ and $\mathrm{cpn}-\beta$. All the mutants are described in detail in Table S2. The thermal stabilities of each mutant were measured in both the apo and ATP-binding states (Fig. S6A and S6B). Notably, in comparison to the wild-type cpn- $\alpha$ and cpn- $\beta$, the N/C-termini deletion mutants exhibited greatly reduced self-assembly ability (Fig. S6C). Markedly, the N/C-termini deletion mutant of cpn- $\alpha$ did not assemble at all.

In comparison with the report that the thermal stability of the thermosome Pf-cpn is related to its C-terminus (Luo and Robb, 2011), our results reveal that the deletion of either the $\mathrm{N}$ - or the C-terminus will reduce the thermal stability of both $\mathrm{cpn}-\alpha$ and cpn- $\beta$ and that both the $\mathrm{N}$ - and $\mathrm{C}$-termini contribute to increasing the overall thermal stability of the thermosomes in either the apo or ATP binding state (Fig. 6A-D). Furthermore, we found that both the $\mathrm{N}$ - and $\mathrm{C}$-termini of $\mathrm{cpn}-\beta$ contribute more to the thermal stability of the thermosome than do the cpn- $\alpha$ termini
(Fig. 6A and 6B). Consistent with the above experiments, ATP binding increases the thermal stability of all the mutants compared to their corresponding apo states without changing their relative thermo-stabilities (Fig. 6C and 6D).

In addition to the interwoven N/C-termini and ATP binding, we sought to determine whether the main structural body (without the N/C-termini) of the thermosome subunit affects its thermal stability. To answer this question, we plotted the thermal stabilities of all variants of $c p n-\alpha$ (Fig. 6E) against the thermal stabilities of all variants of cpn- $\beta$ (Fig. 6E). For each spot, its $X$ value represents the thermal stability of one cpn- $\alpha$ variant, and its $Y$-value represents the thermal stability of the cpn- $\beta$ variant that has the same N/C-termini as the cpn- $\alpha$ variant. We found that all the data points appear slightly above the line $y=x$ and are correlated in a linear form with a normalized correlation coefficient of 0.93 (Fig. 6E). Therefore, whether the main body of the thermosome derives from cpn- $\alpha$ or $\mathrm{cpn}-\beta$, their thermal stabilities are exactly related to their N/C-termini. Although the averaged $3^{\circ} \mathrm{C}$ discrepancy of the thermal stabilities between the cpn- $\alpha$ variant and the corresponding cpn- $\beta$ variant (Fig. $6 \mathrm{E}$ ) represents the slightly higher thermal stability that is contributed by the main body of cpn- $\beta$, this discrepancy is much smaller than what is affected by the different N/C-termini, further emphasizing the important role of the N/C-termini for the thermal stability of thermosomes. In summary, swapping the N/C-termini exactly swaps the thermal stability.

\section{The electrostatic interactions within the interwoven N/C-termini are important for the thermal stability}

To verify that the charged residues of the termini contribute to the thermal stability of the thermosome (Luo and Robb, 2011), we performed $\mathrm{pH}$-dependent thermal stability assays (Figs. 6F and S7). We found that both cpn- $\alpha$ and cpn- $\beta$ have optimal thermal stabilities at $\mathrm{pH}$ values of 7.0-8.0 in either the apo or ATP-binding state and that their thermal stabilities significantly decrease when the $\mathrm{pH}$ value is higher than 9.0 or lower than 6.0. These results can be explained by the electrostatic interactions among the charged residues (Asp, Glu and Lys) of the interwoven N/C-termini, which are responsible for determining the thermal stability of the thermosome. There are three Glu residues and four Lys residues in the cpn- $\alpha$ N/C-termini and five Glu residues, one Asp residue and five Lys residues in the cpn- $\beta \mathrm{N} / \mathrm{C}$-termini (Fig. $5 \mathrm{~A}$ ). Both the net charges of the overall $\mathrm{N} / \mathrm{C}$-termini are close to zero when the $\mathrm{pH}$ value is approximately $7.0-8.0$, and the negatively charged residues can interact with the positively charged residues using their complementary charge. When the $\mathrm{pH}$ value is higher than 9.0 or lower than 6.0, the net charges of the overall N/C-termini are not zero, yielding the electrostatic repulsion within the interwoven termini and thereby reducing the overall thermal stability.

In addition to explaining the $\mathrm{pH}$-dependent thermal stability, the N/C-termini of cpn- $\beta$ have more charged residues (11 in total) than that of cpn- $\alpha$ (7 in total), resulting in a stronger electrostatic interaction within the interwoven termini; thereby, $\mathrm{cpn}-\beta$ has a higher thermal stability compared to $\mathrm{cpn}-\alpha$. 
A

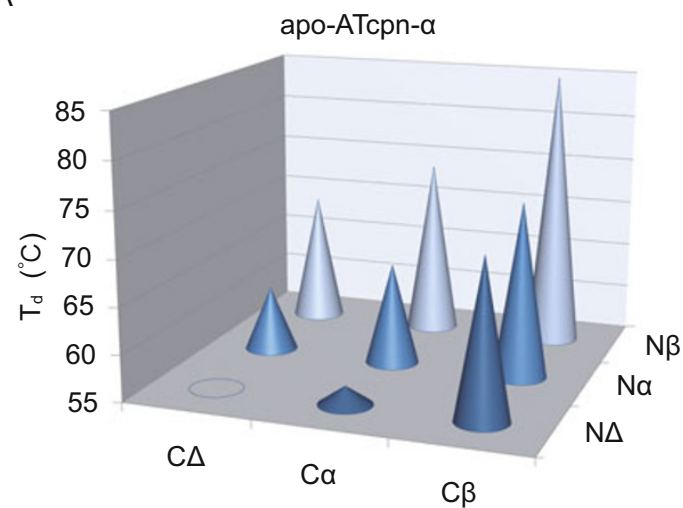

C

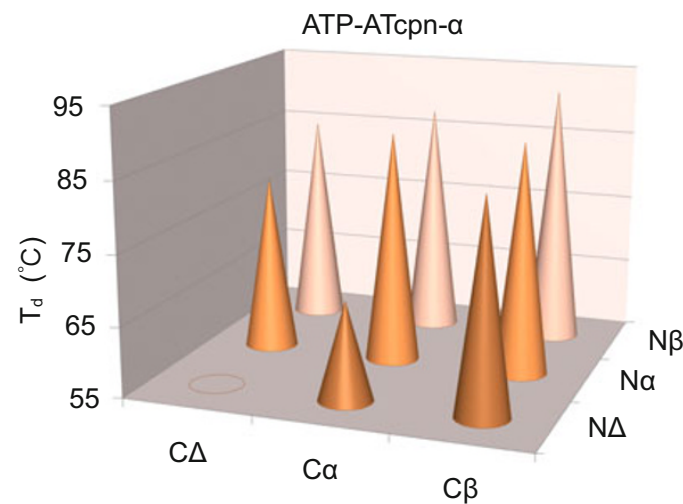

$\mathrm{E}$

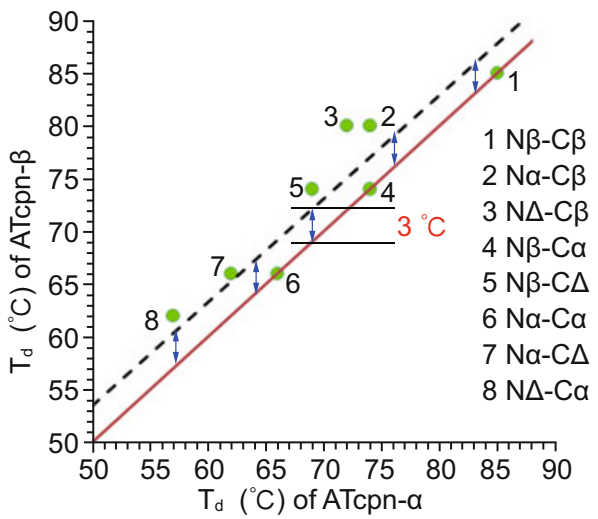

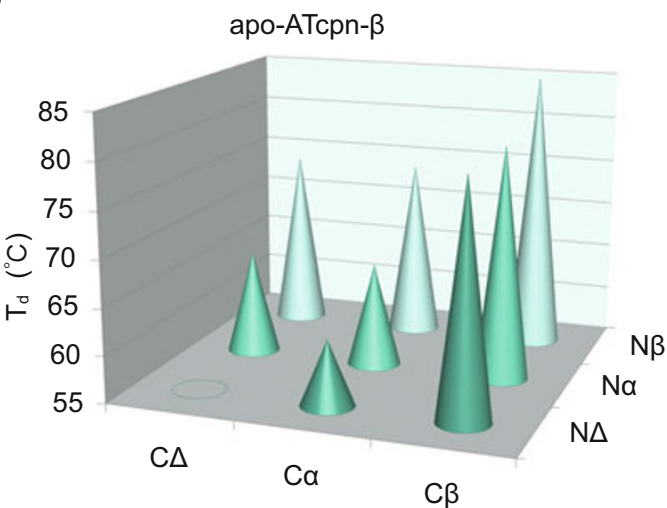

D

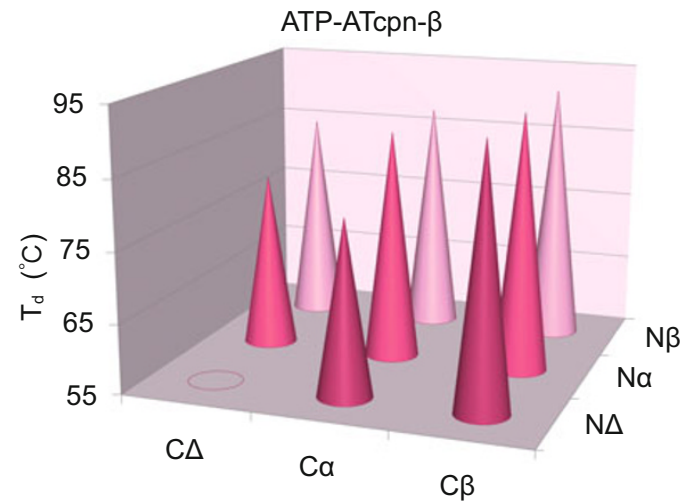

$\mathrm{F}$

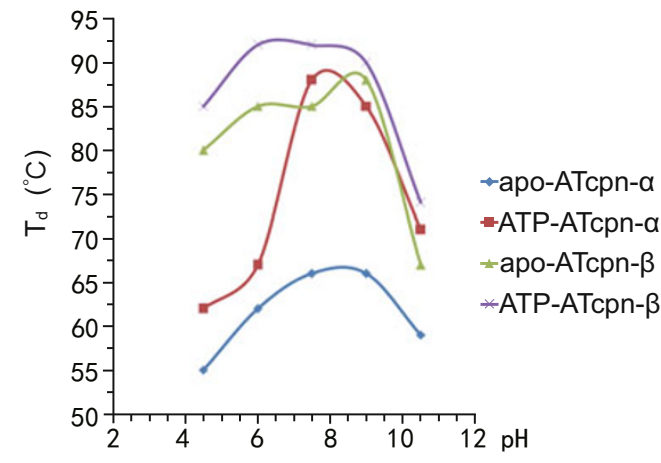

Figure 6. Thermal stabilities of AT thermosome variants created by deleting or swapping the N/C-termini. $(A)$ Plot of $T_{d}$ values of cpn- $\alpha$ variants in the apo state. $\mathrm{C} \Delta, \mathrm{C} \alpha$ and $\mathrm{C} \beta$ in the horizontal axis represent the $\mathrm{C}$-terminus deletion, the $\mathrm{C}$-terminus from cpn- $\alpha$ and the C-terminus from cpn- $\beta$, respectively. $N \Delta, N \alpha$ and $N \beta$ in the vertical axis represent the $N$-terminus deletion, the $N$-terminus from cpn- $\alpha$ and the $\mathrm{N}$-terminus from cpn- $\beta$, respectively. The height of the conical represents the $T_{d}$ value of each cpn- $\alpha$ variant. (B) Plot of $T_{d}$ values of cpn- $\beta$ mutants in the apo state. (C and D) Plot of $T_{d}$ values of $(C) c p n-\alpha$ and (D) cpn- $\beta$ variants in the ATP-binding state. (E) Plot for the correlation between the thermal stabilities of all variants of cpn- $\alpha$ (X-axis) and the thermal stabilities of all variants of cpn- $\beta$ (Y-axis). For each spot, its $X$-value represents the thermal stability of one cpn- $\alpha$ variant, and its $Y$-value represents the thermal stability of the cpn- $\beta$ variant that has the same N/C-termini as the cpn- $\alpha$ variant. $(F)$ The respective $p H$-dependent plots of the $T_{d}$ values of cpn- $\alpha$ and cpn- $\beta$ in the apo and ATP-binding states. See also Figs. S6 and S7.

\section{DISCUSSION}

In the current study, we demonstrated that ATP or its analogs are necessary and sufficient to induce the assembly of thermosomes. We found that the AT thermosome cpn- $\beta$ is more thermally stable than AT cpn- $\alpha$ in both the apo and ATP-binding 


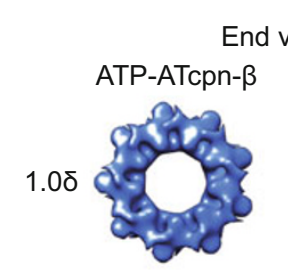

End views
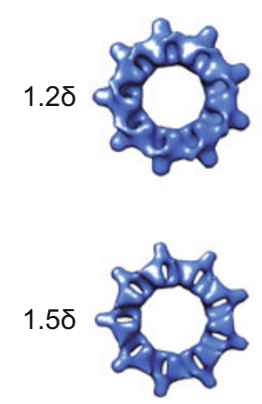
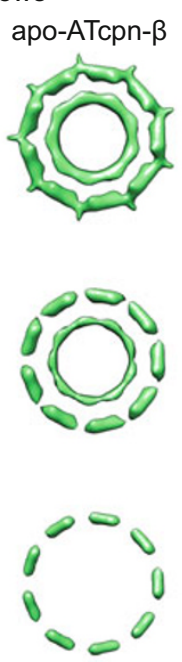

Tilt views $\left(45^{\circ}\right)$

ATP-ATcpn- $\beta$ apo-ATcpn- $\beta$
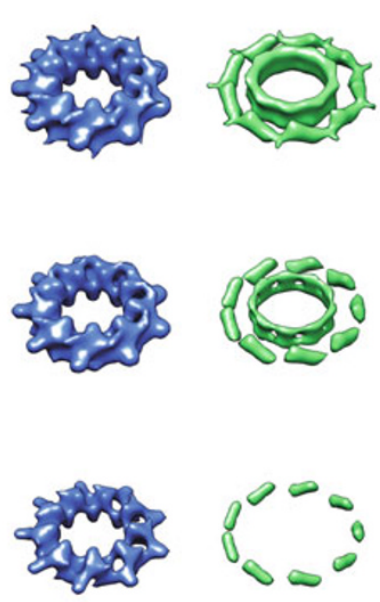

Side views

ATP-ATcpn- $\beta$ apo-ATcpn- $\beta$
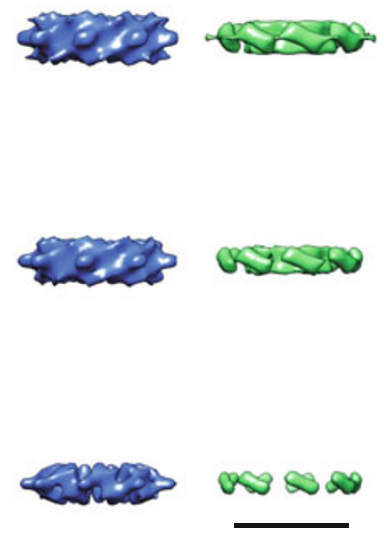

Figure 7. Comparison of the N/C-termini densities between apo cpn- $\beta$ and ATP-bound cpn- $\beta$ from end, tilt and side views at different counter levels. Scale bar is $\mathbf{5 m}$.

states. The thermal stabilities of both thermosomes are significantly enhanced by the addition of ATP, and this enhancement is not dependent on ATP hydrolysis. CryoEM reconstructions of cpn- $\alpha$ and cpn- $\beta$ revealed central densities that account for the interwoven flexible N/C-termini. By combining the structural analysis and biochemical assays, we deduced two important factors that are important for the assembly and thermal stability of thermosomes: one factor is nucleotide binding, and the second, more important, factor is the electrostatic interactions of the interwoven non-conserved flexible N/C-termini.

The thermal stability of group II chaperonins is eventually determined by the summation of the interactions between all the subunits. There are four types of subunit interactions for the apo and ATP-binding states: intra-ring and inter-ring interactions among equatorial domains, intra-ring interactions among lid-apical domains, and interactions among the interwoven termini. Because the intra-ring and inter-ring interactions among equatorial domains are universal in all group II chaperonins and because these two types of interactions involve all conserved residues across chaperonins, they are likely not the dominant factors for the different thermal stabilities between $c p n-\alpha$ and cpn- $\beta$. The intra-ring interactions among lid-apical domains enhance the stability of cpn- $\alpha$ but do not affect that of cpn- $\beta$ (Fig. S4). As a result, the significantly higher thermal stability of cpn- $\beta$ over cpn- $\alpha$ can only be accounted for by their different termini interactions. Both the termini deletion and swapping experiments (Fig. $6 \mathrm{~A}-\mathrm{E}$ ) revealed that $\mathrm{cpn}-\beta$ has favorable N/C-termini that mediate the higher thermal stability of $\mathrm{cpn}-\beta$. The $\mathrm{pH}$-dependent thermal stability assays further suggested that it is the stronger electrostatic interactions of the cpn- $\beta \mathrm{N} / \mathrm{C}$-termini, which contain more charged residues than those of cpn- $\alpha$, that contribute to cpn- $\beta$ 's higher thermal stability compared to cpn- $\alpha$. Notably, the heteromeric thermosome $c p n-\alpha \beta$ has an intermediate thermal stability between cpn- $\alpha$ and $\mathrm{cpn}-\beta$. The explanation for this is that the total number of charged residues from the interwoven N/C-termini of cpn- $\alpha \beta$ is in between that of $c p n-\alpha$ and that of $c p n-\beta$, yielding an intermediate electrostatic interaction and, thereby, an intermediate thermal stability.

From the assembly and thermal stability experiments, we determined that it is the adenosine tail not the phosphate head of ATP that is important for the assembly and stability of thermosomes. Because the ATP-binding pocket is located between the equatorial and intermediate domains, we postulate that the binding of ATP and, more importantly, its adenosine tail will introduce more interactions between the two domains and stabilize their relative motion. Such stabilization would make the interwoven termini more allosterically stable and thereby increase the thermal stability of the entire thermosome. We further compared the central densities of cpn- $\beta$ termini in the apo and ATP-binding states. Both the cryoEM maps of $c p n-\beta$ in the apo and ATP-binding states possess similar central ring-like densities for their N/C-termini. However, after normalizing the cryoEM maps, we found that at each equivalent contour level, the central density in the ATP-bound cpn- $\beta$ was much higher than that in the apo structure (Fig. 7). Such increased central density implies a more stable complex, which is consistent with the above thermal stability assays (Fig. 2F).

Overall, our study provides essential examples for the importance of termini residues for protein assembly and thermal stability.

\section{MATERIALS AND METHODS}

\section{Preparation of AT thermosomes}

Single point mutation D400A, the truncation from 253 to 282 and all sixteen $\mathrm{N}$ - or C-termini deletion and swapping mutants (Table S2) were performed by overlap extension PCR for both AT cpn- $\alpha$ and $c p n-\beta$. For 
$\mathrm{N}$-terminus deletion mutants, the first methionine was preserved due to the needs of protein expression in E.coli. The monomers of AT cpn- $\alpha$ and cpn- $\beta$ as well as all their variants were expressed, purified and assembled into thermosomes as previously reported (Wang et al., 2010).

\section{Thermosome assembly assay}

The monomers of AT cpn- $\alpha$ and cpn- $\beta$ were concentrated and diluted

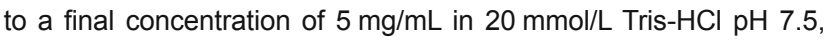
$20 \mathrm{mmol} / \mathrm{L} \mathrm{MgCl}$. Thermosome assembly was performed respectively in the incubation with $2 \mathrm{mmol} / \mathrm{L}$ ATP, ADP, ADP•AIFx, AMP-PNP, GTP, $\mathrm{KH}_{2} \mathrm{PO}_{4}$ and $\left(\mathrm{NH}_{4}\right)_{2} \mathrm{SO}_{4}$ at $37^{\circ} \mathrm{C}$ for $8 \mathrm{~h}$. After incubation, each sample was quickly diluted to $1 \mathrm{mg} / \mathrm{mL}$ and loaded to $4 \%$ native PAGE at $4^{\circ} \mathrm{C}$. The electrophoresis buffer contained $250 \mathrm{mmol} / \mathrm{L}$ Glycine and $25 \mathrm{mmol} / \mathrm{L}$ Tris- $\mathrm{HCl} \mathrm{pH} 8.5$.

\section{Thermo-shift assay}

Thermo-shift experiments were performed according to published methods (Ericsson et al., 2006). Briefly, $5 \mu \mathrm{L} 10 \times$ Sypro Orange (Invitrogen) and $45 \mu \mathrm{L} 0.5 \mathrm{mg} / \mathrm{mL}$ AT cpn monomers were mixed in $0.2 \mathrm{~mL}$ thin-wall PCR tubes (Axygen). The tubes were heated from $25^{\circ} \mathrm{C}$ to $99^{\circ} \mathrm{C}$ in a Roter-Gene 6600 qPCR instrument (Corbett Research, Australia) with an increment of $1^{\circ} \mathrm{C}$. Fluorescence signals were recorded using the green channel of the instrument. The melting temperature, $T_{m}$, was estimated as the temperature corresponding to the minimum of the first derivative of the denaturation curve.

\section{Thermal stability assay}

Thermal stability of all the AT cpns was examined by $4 \%$ native PAGE at $4^{\circ} \mathrm{C}$. The electrophoresis buffer contained $250 \mathrm{mmol} / \mathrm{L}$ Glycine and $25 \mathrm{mmol} / \mathrm{L}$ Tris, $\mathrm{pH} 8.5$. All samples were diluted to $1 \mathrm{mg} / \mathrm{mL}$ in $50 \mathrm{mmol} / \mathrm{L} \mathrm{Tris}-\mathrm{HCl} \mathrm{pH} \mathrm{7.5,} 25 \mathrm{mmol} / \mathrm{L} \mathrm{MgCl}_{2}$ and $100 \mathrm{mmol} / \mathrm{L} \mathrm{KCl}$, with or without $1 \mathrm{mmol} / \mathrm{L}$ ATP. In time-course experiments, each $10 \mu \mathrm{L}$ $1 \mathrm{mg} / \mathrm{mL}$ sample of apo cpn- $\alpha$ was heated to $45^{\circ} \mathrm{C}, 50^{\circ} \mathrm{C}, 55^{\circ} \mathrm{C}$, or $60^{\circ} \mathrm{C}$ for $10-90 \mathrm{~min}$. In temperature gradient experiments, each sample was heated to a certain constant temperature by using PCR instrument (Bio-Rad) for 30 min before native PAGE.

In each lane of native PAGE, the band for the assembly was quantified using TotalLab (http://www.totallab.com/) as C. In the time-course experiments, the relative amount of oligomers $O[t]$ was calculated as $O[t]=C[t] / C[0](t$, time) by using the amount of the oligomer at $0 \mathrm{~min}$ as the reference. Then the relative depolymerized ratio $D[t]=(1-O[t]) 100 \%$ were computed and plotted against heating time. In the temperature gradient experiments, the relative amount of oligomer $A[T]$ was calculated as $A[T]=C[T] / C[R T] \times 100 \%(T$, temperature; RT, room temperature, $25^{\circ} \mathrm{C}$ ) by setting the amount of the oligomer at room temperature as $100 \%$.

In the current study, all the data points regarding to the thermal stabilities were from single experiments.

\section{Cryo-electron microscopy and image processing}

All chaperonins, with and without ATP, were diluted to a final concentration of $3 \mathrm{mg} / \mathrm{mL}$ in $25 \mathrm{mmol} / \mathrm{L}$ Tris- $\mathrm{HCl}(\mathrm{pH} 7.5), 12 \mathrm{mmol} / \mathrm{L} \mathrm{MgCl}_{2}$, $50 \mathrm{mmol} / \mathrm{L} \mathrm{KCl} .3 .5 \mu \mathrm{L}$ of each sample was applied to a 400 -mesh $\mathrm{GiG}^{\mathrm{TM}}$ grid with holes of $2 \mu \mathrm{m}$ diameter separated by $2 \mu \mathrm{m}$. Glow discharge was performed using a JEOL ion sputter JFC-1100 at $0.5 \mathrm{kV}, 5$
$\mathrm{mA}$ for $2 \mathrm{~min}$. Cryo-samples were prepared using a FEI Vitrobot (IV) at $100 \%$ humidity, $25^{\circ} \mathrm{C}$. Low-dose images were captured on a FEI Titan Krios at $300 \mathrm{kV}$ and recorded on a Gatan $4 \mathrm{~K} \times 4 \mathrm{~K} \mathrm{CCD}$ with a final magnification of 160,770 (0.933 $\AA$ /pixel), electron dose $\sim 20$ e/ $/ \AA^{2}$ and defocus between $-1.5 \mu \mathrm{m}$ and $-3.0 \mu \mathrm{m}$. Images were taken manually or using the package Leginon (Suloway et al., 2005).

All AT cpn particles were automatically picked using a GPUaccelerated program GAutoMatch developed in our lab. The defocus value of each micrograph was determined by CTFFIND3 (Mindell and Grigorieff, 2003) and the CTF of each particle was corrected using "applyctf" in EMAN (Ludtke et al., 1999). The reference-free twodimensional classification was performed using "refine2d.py" in EMAN. Initial models were generated by filtering previous 9-fold cryoEM maps of AT cpn- $\beta$ (Huo et al., 2010) to $30 \AA$ resolution. Refinements or multireference refinement were performed using "refine" or "multirefine" in EMAN with SPIDER scripts embedded for correspondence analysis (CORAN) of each image class (Frank et al., 1996; Shaikh et al., 2008). The final reconstructed map was sharpened by an amplitude correction algorithm in BFACTOR (from Niko Grigorieff's lab). CryoEM maps were segmented, displayed and fitted with atomic models using UCSF Chimera (Pettersen et al., 2004).

The statistics of cryoEM reconstructions of all thermosomes are summarized in Table S1.

\section{Symmetry-restrained molecular dynamics flexible fitting}

MDFF is a computational method to fit atomic models into cryo-EM density maps by molecular dynamics simulations (Trabuco et al., 2008; Trabuco et al., 2011). An extension of the method, namely symmetryrestrained MDFF, is developed recently to incorporate structural symmetry information into the fitting procedure (Chan et al., 2011). The initial atomic model of AT cpn- $\beta$ was obtained from its crystal structure (Huo et al., 2010) (PDB code, 3KO1); the one of AT cpn- $\alpha$ was built based on its high-resolution cryoEM density. After rigid body docking into the cryoEM map, the proteins were solvated in a box of water molecules with $150 \mathrm{mmol} / \mathrm{L} \mathrm{NaCl}$ in VMD (Humphrey et al., 1996), using $15 \AA$ of padding in all directions. Extra ions were added to neutralize the systems. The simulations were performed with program NAMD 2.8 (Phillips et al., 2005), using the CHARMM27 force field with CMAP corrections (MacKerell et al., 1998; Mackerell et al., 2004). All the fitting procedure is the same as the application of symmetry-restrained MDFF to chaperonin reported previously (Chan et al., 2011).

\section{ACCESSION NUMBERS}

All cryo-electron microscopy density maps in this paper are deposited in the Electron Microscopy Data Bank (EMDB) and their corresponding atomic models fitted by MDFF are deposited in the Protein Data Bank (PDB). The entry numbers (EMDB/PDB) for 8-fold and 9-fold apo cpn- $\alpha$, and cpn- $\beta$ in apo and ATP-binding state are EMD_5391/3J1B, EMD_5392/3J1C, EMD_5395/3J1E, EMD_5396/3J1F, respectively. See also Table S1.

\section{ACKNOWLEDGEMENTS}

We would like to thank Gang Ji (CBI, IBP) and Bingquan Gao (FS group) for the help with data collection, and Zhongjun $\mathrm{Hu}$ and Xue Wang (FS group) for data processing. This work was supported by 
grants the National Basic Research Program (973 Program) (No. 2011CB910303), the Chinese Academy of Sciences (KGCX1-YW-13, and KSCX2-EW-J-3) and the National Natural Science Foundation of China (Grant Nos. 31170700 and 31021062), and a grant to K.S. from the National Institutes of Health (9P41GM104601). K.Z. (F.S. group) was supported by a Gatan China Scholarship (2011-2012). All the cryoEM datasets were collected at the Center for Biological Imaging, Core Facility for Protein Sciences, Chinese Academy of Sciences. Computer time for MDFF was provided through grant MCA93S028 from the Extreme Science and Engineering Discovery Environment (XSEDE), which is supported by National Science Foundation grant OCl-1053575.

\section{ABBREVIATIONS}

AT, Acidianus tengchongensis; cryoEM, cryo-electron microscopy; cpn, chaperonin; MDFF, molecular dynamics flexible fitting; PAGE, polyacrylamide gel electrophoresis

\section{COMPLIANCE WITH ETHICS GUIDELINES}

Kai Zhang, Li Wang, Yanxin Liu, Kwok-Yan Chan, Xiaoyun Pang, Klaus Schulten, Zhiyang Dong and Fei Sun declare that they have no conflict of interest.

This article does not contain any studies with human or animal subjects performed by the any of the authors.

\section{REFERENCES}

Bigotti, M.G., and Clarke, A.R. (2008). Chaperonins: The hunt for the Group II mechanism. Archi Biochem Biophy 474, 331-339.

Bukau, B., and Horwich, A.L. (1998). The Hsp70 and Hsp60 chaperone machines. Cell 92, 351-366.

Chan, K.Y., Gumbart, J., McGreevy, R., Watermeyer, J.M., Sewell, B.T., and Schulten, K. (2011). Symmetry-restrained flexible fitting for symmetric EM maps. Structure 19, 1211-1218.

Clare, D.K., Bakkes, P.J., van Heerikhuizen, H., van der Vies, S.M., and Saibil, H.R. (2009). Chaperonin complex with a newly folded protein encapsulated in the folding chamber. Nature 457, 107-110.

Clare, D.K., Stagg, S., Quispe, J., Farr, G.W., Horwich, A.L., and Saibil, H.R. (2008). Multiple states of a nucleotide-bound group 2 chaperonin. Structure 16, 528-534.

Clare, D.K., Vasishtan, D., Stagg, S., Quispe, J., Farr, G.W., Topf, M., Horwich, A.L., and Saibil, H.R. (2012). ATP-triggered conformational changes delineate substrate-binding and -folding mechanics of the GroEL chaperonin. Cell 149, 113-123.

Dekker, C., Roe, S.M., McCormack, E.A., Beuron, F., Pearl, L.H., and Willison, K.R. (2011). The crystal structure of yeast CCT reveals intrinsic asymmetry of eukaryotic cytosolic chaperonins. EMBO J 30, 3078-3090.

Ditzel, L., Lowe, J., Stock, D., Stetter, K.O., Huber, H., Huber, R., and Steinbacher, S. (1998). Crystal structure of the thermosome, the archaeal chaperonin and homolog of CCT. Cell 93, 125-138.

Douglas, N.R., Reissmann, S., Zhang, J., Chen, B., Jakana, J., Kumar, R., Chiu, W., and Frydman, J. (2011). Dual action of ATP hydrolysis couples lid cosure to substrate release into the group II chaperonin chamber. Cell 144, 240-252.

Ellis, R.J. (2006). Protein folding: inside the cage. Nature 442, 360-362.
Ericsson, U.B., Hallberg, B.M., Detitta, G.T., Dekker, N., and Nordlund, P. (2006). Thermofluor-based high-throughput stability optimization of proteins for structural studies. Anal Biochem 357, 289-298.

Frank, J., Radermacher, M., Penczek, P., Zhu, J., Li, Y., Ladjadj, M., and Leith, A. (1996). SPIDER and WEB: processing and visualization of images in 3D electron microscopy and related fields. J Struct Biol 116, 190-199.

Frydman, J., Nimmesgern, E., Erdjument-Bromage, H., Wall, J.S., Tempst, P., and Hartl, F.U. (1992). Function in protein folding of TRiC, a cytosolic ring complex containing TCP-1 and structurally related subunits. EMBO J 11, 4767-4778.

Gao, Y., Thomas, J.O., Chow, R.L., Lee, G.H., and Cowan, N.J. (1992). A cytoplasmic chaperonin that catalyzes beta-actin folding. Cell 69, 1043-1050

He, Z.G., Zhong, H., and Li, Y. (2004). Acidianus tengchongensis sp. nov., a new species of acidothermophilic archaeon isolated from an acidothermal spring. Curr Microbiol 48, 159-163.

Humphrey, W., Dalke, A., and Schulten, K. (1996). VMD: visual molecular dynamics. J Mol Graph 14, 33-38, 27-38.

Huo, Y., Hu, Z., Zhang, K., Wang, L., Zhai, Y., Zhou, Q., Lander, G., Zhu, J., He, Y., Pang, X., et al. (2010). Crystal structure of group II chaperonin in the open state. Structure 18, 1270-1279.

Izumi, M., Fujiwara, S., Takagi, M., Fukui, K., and Imanaka, T. (2001). Two kinds of archaeal chaperonin with different temperature dependency from a hyperthermophile. Biochem Biophys Res Commun 280, 581-587.

Kagawa, H.K., Yaoi, T., Brocchieri, L., McMillan, R.A., Alton, T., and Trent, J.D. (2003). The composition, structure and stability of a group II chaperonin are temperature regulated in a hyperthermophilic archaeon. Mol Microbiol 48, 143-156.

Kim, S., Willison, K.R., and Horwich, A.L. (1994). Cystosolic chaperonin subunits have a conserved ATPase domain but diverged polypeptide-binding domains. Trends Biochem Sci 19, 543-548.

Klumpp, M., Baumeister, W., and Essen, L.O. (1997). Structure of the substrate binding domain of the thermosome, an archaeal group II chaperonin. Cell 91, 263-270.

Ludtke, S.J., Baldwin, P.R., and Chiu, W. (1999). EMAN: semiautomated software for high-resolution single-particle reconstructions. J Struct Biol 128, 82-97.

Luo, H., and Robb, F.T. (2011). A modulator domain controlling thermal stability in the Group II chaperonins of Archaea. Archi Biochem Biophy 512, 111-118.

MacKerell, A.D., Bashford, D., Bellott, Dunbrack, R.L., Evanseck, J.D., Field, M.J., Fischer, S., Gao, J., Guo, H., Ha, S., et al. (1998). Alltom empirical potential for molecular modeling and dynamics studies of proteins. J Phys Chem 102, 3586-3616.

Mackerell, A.D., Jr., Feig, M., and Brooks, C.L., 3rd (2004). Extending the treatment of backbone energetics in protein force fields: limitations of gas-phase quantum mechanics in reproducing protein conformational distributions in molecular dynamics simulations. J Comput Chem 25, 1400-1415.

Mindell, J.A., and Grigorieff, N. (2003). Accurate determination of local defocus and specimen tilt in electron microscopy. J Struct Biol 142, 334-347.

Munoz, I.G., Yebenes, H., Zhou, M., Mesa, P., Serna, M., Park, A.Y., Bragado-Nilsson, E., Beloso, A., de Carcer, G., Malumbres, M., et al. (2011). Crystal structure of the open conformation of the mam- 
malian chaperonin CCT in complex with tubulin. Nat Struct Mol Biol 18, 14-19.

Nitsch, M., Klumpp, M., Lupas, A., and Baumeister, W. (1997). The thermosome: alternating alpha and beta-subunits within the chaperonin of the archaeon Thermoplasma acidophilum. J Mol Biol 267, 142-149.

Pettersen, E.F., Goddard, T.D., Huang, C.C., Couch, G.S., Greenblatt, D.M., Meng, E.C., and Ferrin, T.E. (2004). UCSF Chimera--a visualization system for exploratory research and analysis. J Comput Chem 25, 1605-1612.

Phillips, J.C., Braun, R., Wang, W., Gumbart, J., Tajkhorshid, E., Villa, E., Chipot, C., Skeel, R.D., Kale, L., and Schulten, K. (2005). Scalable molecular dynamics with NAMD. J Comput Chem 26, 1781-1802.

Phipps, B.M., Hoffmann, A., Stetter, K.O., and Baumeister, W. (1991). A novel ATPase complex selectively accumulated upon heat shock is a major cellular component of thermophilic archaebacteria. EMBO J 10, 1711-1722.

Ranson, N.A., Clare, D.K., Farr, G.W., Houldershaw, D., Horwich, A.L., and Saibil, H.R. (2006). Allosteric signaling of ATP hydrolysis in GroEL-GroES complexes. Nat Struct Mol Biol 13, 147-152.

Ranson, N.A., Farr, G.W., Roseman, A.M., Gowen, B., Fenton, W.A., Horwich, A.L., and Saibil, H.R. (2001). ATP-bound states of GroEL captured by cryo-electron microscopy. Cell 107, 869-879.

Sahlan, M., Kanzaki, T., and Yohda, M. (2009). Construction and characterization of the hetero-oligomer of the group II chaperonin from the hyperthermophilic archaeon, Thermococcus sp. strain KS-1. Extremophiles 13, 437-445.

Shaikh, T.R., Gao, H., Baxter, W.T., Asturias, F.J., Boisset, N., Leith, A., and Frank, J. (2008). SPIDER image processing for single-particle reconstruction of biological macromolecules from electron micrographs. Nat Protoc 3, 1941-1974.

Shomura, Y., Yoshida, T., lizuka, R., Maruyama, T., Yohda, M., and Miki, K. (2004). Crystal structures of the group II chaperonin from Thermococcus strain KS-1: steric hindrance by the substituted amino acid, and inter-subunit rearrangement between two crystal forms. J Mol Biol 335, 1265-1278.

Suloway, C., Pulokas, J., Fellmann, D., Cheng, A., Guerra, F., Quispe,
J., Stagg, S., Potter, C.S., and Carragher, B. (2005). Automated molecular microscopy: the new Leginon system. J Struct Biol 151, 41-60.

Tilly, K., Murialdo, H., and Georgopoulos, C. (1981). Identification of a second Escherichia coli groE gene whose product is necessary for bacteriophage morphogenesis. Proc Natl Acad Sci U S A 78, 1629-1633.

Trabuco, L.G., Schreiner, E., Gumbart, J., Hsin, J., Villa, E., and Schulten, K. (2011). Applications of the molecular dynamics flexible fitting method. J Struct Biol 173, 420-427.

Trabuco, L.G., Villa, E., Mitra, K., Frank, J., and Schulten, K. (2008). Flexible fitting of atomic structures into electron microscopy maps using molecular dynamics. Structure 16, 673-683.

Wang, L., Hu, Z.J., Luo, Y.M., Huo, Y.W., Ma, Q., He, Y.Z., Zhang, Y.Y., Sun, F., and Dong, Z.Y. (2010). Distinct symmetry and limited peptide refolding activity of the thermosomes from the acidothermophilic archaea Acidianus tengchongensis S5(T). Biochem Biophy Res Commun 393, 228-234.

Xu, Z., Horwich, A.L., and Sigler, P.B. (1997). The crystal structure of the asymmetric GroEL-GroES-(ADP)7 chaperonin complex. Nature 388, 741-750.

Yoshida, T., Ideno, A., Hiyamuta, S., Yohda, M., and Maruyama, T. (2001). Natural chaperonin of the hyperthermophilic archaeum, Thermococcus strain KS-1: a hetero-oligomeric chaperonin with variable subunit composition. Mol Microbiol 39, 1406-1413.

Yoshida, T., Ideno, A., Suzuki, R., Yohda, M., and Maruyama, T. (2002). Two kinds of archaeal group II chaperonin subunits with different thermostability in Thermococcus strain KS-1. Mol Microbiol 44, 761-769.

Yoshida, T., Kanzaki, T., lizuka, R., Komada, T., Zako, T., Suzuki, R., Maruyama, T., and Yohda, M. (2006). Contribution of the C-terminal region to the thermostability of the archaeal group II chaperonin from Thermococcus sp. strain KS-1. Extremophiles 10, 451-459.

Zhang, J., Baker, M.L., Schroder, G.F., Douglas, N.R., Reissmann, S., Jakana, J., Dougherty, M., Fu, C.J., Levitt, M., Ludtke, S.J., et al. (2010). Mechanism of folding chamber closure in a group II chaperonin. Nature 463, 379-383. 